'Овчинников B. A. (luch.d.grmu@mail.ru),2Довнар O.C. (Odovn@mail.ru),

'УО «Гродненский государственный медицинский университет», Гродно, Беларусь 2 У3 «Гродненская областная клиническая больница», Гродно, Беларусь

Введение. Эффективность сочетанной лучевой терапии с применением высокодозной брахитерапии при раке предстательной железы остается недостаточно изученной.

Цель работы. Оченить эффективность сочетанной лучевой терапии рака предстательной железыс андрогенной деприваџией в условиях эскалащии разовой дозы брахитерапии (8,5 Гр и 9,5 Гр).

Материал и методы. Наблюдения проведены у 75 пациентов с местно-распространенным (ТЗа) и локализованным раком предстательной железы с неблагоприятным прогнозом.

Результаты и обсуждение. Эскалащия дозы брахитерапии с 8,5 Гр до 9,5 Гр (две имплантаџии) и андрогенная депривация улучшают результаты лучевой терапии: 100\% биохимический контроль в течение двух лет.

Вывод. Эффективность лечения рака предстательной железы увеличивается при эскалации дозы сочетанной лучевой терапии и андрогенной деприваџии.

Ключевые слова: сочетанная лучевая терапия, рак предстательной железы, андрогенная депривация.

\section{Введение}

По темпам прироста рак предстательной железы (РПЖ) занимает первое место среди всех злокачественных новообразований в Республике Беларусь [1]. В настоящее время наблюдается экспоненциальный рост заболеваемости РПЖ. Показатели смертности также остаются высокими. За последние десятилетия смертность от РПЖ выросла почти в 3 раза (с 7,0 случая на 100 тыс. мужского населения в 1990 г. до 19,5 на 100 тыс. населения в 2011 г.) [2].

Способы радикального лечения локализованного РПЖ - простатэктомия, дистанционная лучевая терапия, брахитерапия (внутритканевая лучевая терапия) [3, 4]. В связи с многообразием методов лечения рака предстательной железы актуальна проблема выбора конкретной технологии лечения, оценки ее эффективности. Одним из современных способов лечения РПЖ является сочетанная лучевая терапия (СЛТ) с применением контактного облучения в виде высокодозной брахитерапии (ВДБ) и дистанционной лучевой терапии (ДЛТ). ВДБ РПЖ имеет преимущества перед другими методами в связи с избирательным подведением дозы излучения к облучаемому объему, включающему макро- и микроскопические проявления злокачественной опухоли $[3,4,5]$. Результаты СЛТ РПЖ сопоставимы с результатами простатэктомии и превышают ДЛТ, вместе с тем наилучший режим облучения при помощи ВДБ остается неизвестным $[3,6]$. Андрогенная депривация (АД) улучшала результаты ДЛТ по критерию пятилетней выживаемости $[7,8]$. Имеются данные, что АД улучшает результаты СЛТ с ВДБ, но роль АД в этих условиях нуждается в дальнейшем изучении [6].

Цель работы: оценить биологическую эффективность дозы при сочетанной лучевой терапии рака предстательной железы с андрогенной депривацией в условиях эскалации разовой дозы брахитерапии (8,5 Гр и 9,5 Гр).

\section{Материал и методы}

Проведены наблюдения у 75 пациентов с РПЖ. Возраст - 51-77 лет. Концентрация общего простатспецифического антигена (ПСА) в сыворотке крови определялась методом твердофазного иммуноферментного анализа [9].

Определялся уровень ПСА до лечения и через $3,6,12,24$ месяца после окончания СЛТ. Выполнялись также и другие исследования в соответствии с рекомендациями, изложенными в «Алгоритмах диагностики и лечения злокачественных образований. Минск, 2012» [10]. У всех пациентов диагноз верифицирован морфологически аденокарцинома. Локализованный РПЖ (T2a-c) наблюдался в 58 случаях, местно-распространенный - в 17 (Т3a). Во всех случаях локализованный рак был с неблагоприятным прогнозом (стадия Т2с или сумма Глисона $>7$, или ПСА $>20$ нг/мл). Всем пациентам проводилась телегамматерапия (ТГТ) на область предстательной железы и семенных пузырьков, лимфатических узлов таза в режиме обычного фракционирования дозы до суммарной дозы 40-44 Гр (средняя суммарная доза 40,8 $\pm 0,2$ Гр). ВДБ с иридием 192 проводилась в виде двух имплантаций по 8,5 Гр или 9,5 Гр на предстательную железу до или после ТГТ. Общая длительность СЛТ составляла 54-150 дней, в среднем 82,9 $\pm 1,7$ дня. Все пациенты получали адъювантную гормонотерапию: флутамид - по 0,25 три раза в день внутрь. 15 пациентам СЛТ применялась с ВДБ в разовой дозе 8,5 Гр (1-я группа). 21 пациенту СЛТ использовалась с ВДБ в разовой дозе 9,5 Гр (2-я группа). Пациенты 1-й и 2-й групп отказались от орхиэктомии. 24 пациента после андрогенной депривации (двусторонняя орхиэктомия) получали СЛТ с ВДБ в разовой дозе 8,5 Гр (третья группа). 15 пациентам после двусторонней орхиэктомии применялась СЛТ с ВДБ в разовой дозе 9,5 Гр (4-я группа). 
В 1-й группе количество пациентов с локализованным РПЖ неблагоприятного прогноза составило $80,0 \%(\mathrm{n}=12)$, с местно-распространенным РПЖ - 20,0\% $(n=3)$, во 2-й - 85,6 $(n=18)$ и $14,3 \%(n=3)$, соответственно. В 3-й группе количество пациентов с локализованным РПЖ неблагоприятного прогноза было равным $75,0 \%$ $(\mathrm{n}=12)$, с местнораспространенным РПЖ $-25,0 \%$ $(n=6)$, в 4-й - 66,7 $(n=15)$ и $33,3 \%(n=6)$, соответственно. Таким образом, во всех группах преобладали пациенты с неблагоприятным прогнозом. Возраст пациентов, длительность лечения в рассматриваемых группах также не различались статистически достоверно. нятой классификации [11].

Статистический анализ проводился с помо2010 и программы «RStudio 1.1.383». Использовалась парная статистика (Стьюдента). Численные данные приведены в форме: среднеарифметическое $(\mathrm{M}) \pm$ среднеарифметическая ошибка (m).

Проводились также попарные сравнения долей контроля между группами. Так как в некоторых случаях теоретические частоты были менее 5, кроме того, встречались частоты, равные 0 , то сравнение проводилось с использованием теста Бошлу [12]; р-значения результатов попарных сравнений долей были скорректированы с использованием поправки Холма-Бонферрони [13].

\section{Результаты и обсуждение}

Динамика концентрации ПСА при СЛТ и андрогенной депривации представлена в таблице.
Лучевые реакции оценивались по общеприщью программного пакета Microsoft Office Excel

Из представленных в таблице данных следует, что во всех группах после окончания лечения наступало резкое статистически достоверное снижение уровня ПСА. Исходная концентрация ПСА перед началом лечения в исследуемых группах статистически достоверно не различалась.

Через $3,6,12,18,24$ месяца после окончания лечения различия в концентрации ПСА в 1-й и 2-й группах не были статистически достоверными $(\mathrm{p}>0,05)$.

Концентрация ПСА в 3-й группе после СЛТ была существенно ниже по сравнению с аналогичным показателем в 1-й и во 2-й группах во все сроки исследований, за исключением срока в 24 месяца относительно 2-й группы.

В 4-й группе, в отличие от 3-й, после СЛТ наблюдалось статистически достоверное снижение концентрации ПСА относительно уровня после АД ( $<<0,01)$. Через 3, 6, 12, 18, 24 месяца после окончания лечения концентрация ПСА в 4-й группе была существенно ниже, чем в 1-й и 2-й группах, за исключением срока в 12 месяцев относительно 2-й группы.

Биохимический контроль в течение двух лет в 1-й группе составил 7 случаев $(46,7)$. Биохимический контроль означает отсутствие биохимического рецидива, то есть превышения надира (плато ПСА после первоначального снижения на фоне терапии) на 2,0 нг/мл и более $[9,12]$. Рост ПСА, или биохимический рецидив, свидетельствует об активизации болезни и предшествует ее прогрессированию $[10,14]$.

Во 2-й группе биохимический контроль в течение двух лет был у 17 пациентов $(81,0 \%)$, что больше (в 1,7 раза), чем в 1-й группе, но статистически не достоверно.

В 3-й группе биоTaблица. - Динамика концентрации ПСА (нг/мл) при СЛТ и андрогенной химический контроль депривации

\begin{tabular}{|c|c|c|c|c|}
\hline $\begin{array}{c}\text { Сроки } \\
\text { исследования }\end{array}$ & 1-я группа & 2-я группа & 3-я группа & 4-я группа \\
\hline До лечения & $14,1 \pm 2,2$ & $20,5 \pm 2,3$ & $19,7 \pm 3,5$ & $23,3 \pm 5,4$ \\
\hline $\begin{array}{c}\text { После андрогенной } \\
\text { депривации }\end{array}$ & - & - & $1,4 \pm 0,8 \mathrm{p}<0,001 \dagger$ & $2,9 \pm 0,8 \mathrm{p}<0,001 \dagger$ \\
\hline $\begin{array}{c}3 \text { месяца после } \\
\text { СЛТ }\end{array}$ & $\begin{array}{c}3,0 \pm 1,0 \\
\mathrm{p}<0,001 \dagger\end{array}$ & $3,1 \pm 1,1 \mathrm{p}<0,001 \dagger$ & $\begin{array}{c}0,2 \pm 0,03 \mathrm{p}<0,001 \dagger \\
\mathrm{i}_{\mathrm{p}<0,05^{\mathrm{i}}} \\
\mathrm{p}<0,05^{*}\end{array}$ & $\begin{array}{c}0,5 \pm 0,3 \mathrm{p}<0,001 \dagger \\
\mathrm{p}<0,05^{\mathrm{i}} \\
\mathrm{p}<0,05^{*}\end{array}$ \\
\hline $\begin{array}{c}6 \text { месяцев после } \\
\text { СЛТ }\end{array}$ & $\begin{array}{c}2,0 \pm 0,6 \\
\mathrm{p}<0,001 \dagger\end{array}$ & $1,2 \pm 0,3 \mathrm{p}<0,001 \dagger$ & $\begin{array}{c}0,2 \pm 0,05 \mathrm{p}<0,001 \dagger \\
\mathrm{p}<0,01^{i} \\
\mathrm{p}<0,01^{*}\end{array}$ & $\begin{array}{c}0,3 \pm 0,08 \mathrm{p}<0,001 \dagger \\
\mathrm{p}<0,01^{i} \\
\mathrm{p}<0,01^{*}\end{array}$ \\
\hline $\begin{array}{c}12 \text { месяцев после } \\
\text { СЛТ }\end{array}$ & $\begin{array}{c}1,9 \pm 0,5 \\
\mathrm{p}<0,001 \uparrow\end{array}$ & $0,9 \pm 0,2 \mathrm{p}<0,001 \dagger$ & $\begin{array}{c}0,1 \pm 0,04 \mathrm{p}<0,001 \dagger \\
\mathrm{p}<0,001^{\mathrm{i}} \\
\mathrm{p}<0,001^{*}\end{array}$ & $\begin{array}{c}0,5 \pm 0,2 \mathrm{p}<0,001 \dagger \\
\mathrm{p}>0,05^{\mathrm{i}} \\
<0,001^{*}\end{array}$ \\
\hline $\begin{array}{l}18 \text { месяцев } \\
\text { после СЛТ }\end{array}$ & $\begin{array}{c}3,6 \pm 0,9 \\
\mathrm{p}<0,001 \dagger\end{array}$ & $3,1 \pm 1,1 \mathrm{p}<0,001 \dagger$ & $\begin{array}{c}0,4 \pm 0,2 \\
\mathrm{p}<0,001 \dagger \\
\mathrm{p}<0,05^{i} \\
\mathrm{p}<0,001^{*}\end{array}$ & $\begin{array}{c}0,5 \pm 0,1 \mathrm{p}<0,001 \dagger \\
\mathrm{p}<0,05^{\mathrm{i}} \\
\mathrm{p}<0,01^{*}\end{array}$ \\
\hline $\begin{array}{c}24 \text { месяца после } \\
\text { СЛТ }\end{array}$ & $\begin{array}{c}3,8 \pm 1,0 \\
\mathrm{p}<0,001 \dagger\end{array}$ & $4,3 \pm 1,9 \mathrm{p}<0,001 \dagger$ & $\begin{array}{c}0,8 \pm 0,4 \\
\mathrm{p}<0,001^{\dagger} \\
\mathrm{p}>0,05^{i} \\
\mathrm{p}<0,01^{*}\end{array}$ & $\begin{array}{c}0,3 \pm 0,07 \mathrm{p}<0,001 \dagger \\
\mathrm{p}<0,05^{\mathrm{i}} \\
\mathrm{p}<0,01^{*}\end{array}$ \\
\hline
\end{tabular}

Примечание: $p$ - критерий достоверности различий: *- относительно 1-й группьг; ${ }^{i}$ - относительно 2-й группь;; †-относительно исходного уровня в период (два года после СЛТ) наблюдался у 22 пациентов $(91,7 \%$, $\mathrm{p}<0,0087$ ), что превышало аналогичный показатель в 1-й группе

Наибольший уровень биохимического контроля отмечен в 4-й группе - у 15 пациентов $(100 \%, \mathrm{p}<0,0044)$, что также превышало аналогичный показатель в 1-й группе (рис.).

Повышение эффективности лечения при СЛТ в комплексе с андрогенной депривацией связано с ликвидацией при антиандрогенной терапии андрогенного стимула клеток предстательной железы, что приводит к их апоптозу [15]. (в 2,0 раза). 
Биохимический контроль при СЛТ

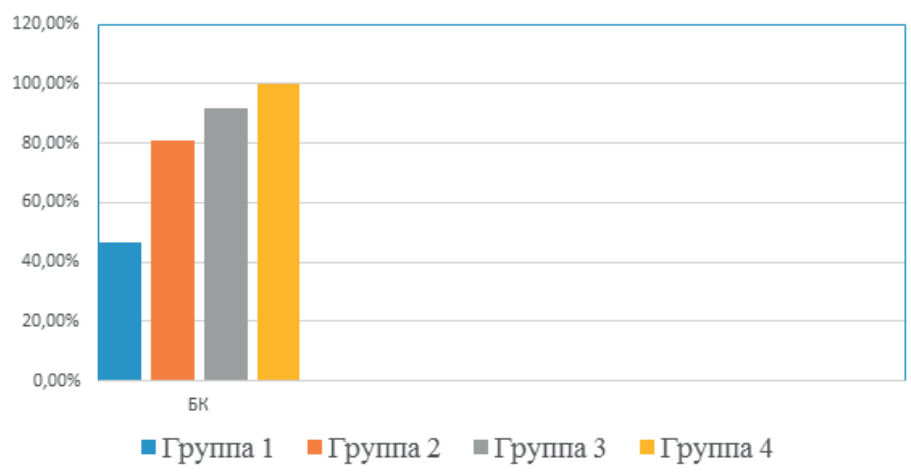

Рисунок. - Частота биохимического контроля через 24 месяца после СЛТ (в \%)

Лучевые реакции первой степени со стороны мочевыделительной системы были в 1-й группе в 4 наблюдениях и в 4 случаях достигали 2-й степени, всего в этой группе местные лучевые реакции со стороны мочевыделительной системы наблюдались у 8 пациентов (53,3\%).

Первая степень лучевых реакций со стороны мочевыделительной системы (учащенное мочеиспускание или никтурия в 2 раза более чем исходные; упорная дизурия, не требующая лекарственной терапии) [11].

Вторая степень лучевых реакций со стороны мочевыделительной системы (мочеиспускание или никтурия не чаще 1 раза в час). Упорная дизурия, спазмы мочевого пузыря, требующие назначения локальных анестетиков (например пиридиума) [11].

Со стороны кишечника в 1-й группе лучевые реакции были первой степени в 9 наблюдениях, второй степени - в одном случае. Всего лучевые реакции со стороны кишечника отмечены в 10 наблюдениях (66,7\%).

Первая степень лучевых реакций со стороны кишечника - это возросшая частота или изменения в качестве кишечных отправлений, не требующие назначения медикаментов / ректальный дискомфорт, не требующий анальгетиков [11].

Вторая степень лучевых реакций со стороны кишечника - диарея, требующая назначения парасимпатолитиков (например ломотил); слизистые выделения, не требующие санитарных прокладок; ректальные или абдоминальные боли, требующие назначения анальгетиков [11].

Во 2-й группе лучевые реакции первой степени со стороны мочевыделительной системы имелись у 17 пациентов (в 4 случаях - второй степени). Во 2-й группе лучевые реакции со стороны мочевыделительной системы выявлены во всех случаях (100\%). Наблюдалось статистически достоверное увеличение частоты лучевых реакций (1-й и 2-й степеней) со стороны мочевыделительной системы во 2-й группе ( $<<0,0058)$. Лучевые реакции кишечника первой степени имелись у 13 пациентов и второй степени - у 2. Всего лучевые реакции со стороны кишечника были в этой группе в 15 наблюдениях $(71,4 \%)$.
Не отмечено статистически достоверного увеличения лучевых реакций со стороны кишечника во 2-й группе.

В 3-й группе лучевые реакции первой степени со стороны мочевыделительной системы были у 12 пациентов, в 5 случаях - второй степени. В 3-й группе лучевые реакции со стороны мочевыделительной системы имелись у 17 пациентов $(80,0 \%)$. Лучевые реакции кишечника первой степени были у 8 пациентов и второй степени - у 3. Всего лучевые реакции первой и второй степеней со стороны кишечника были в этой группе в 11 наблюдениях (45,8\%). Имелось статистически достоверное увеличение частоты лучевых реакций со стороны мочевыделительной системы во 2-й группе относительно 3-й группы $(\mathrm{p}<0,0267)$. Вместе с тем не было статистически достоверных изменений со стороны частоты лучевых реакций кишечника.

В 4-й группе лучевые реакции первой степени со стороны мочевыделительной системы были у 14 пациентов, в 1 случае - второй степени. В 4-й группе лучевые реакции со стороны мочевыделительной системы выявлены во всех случаях (100\%). Лучевые реакции кишечника первой степени имелись у 9 пациентов и второй степени - у 2. Всего лучевые реакции первой и второй степеней были в этой группе в 11 наблюдениях $(73,3 \%)$. Установлено статистически достоверное увеличение частоты лучевых реакций со стороны мочевыделительной системы в 4-й группе относительно 1-й группы ( $<<0,0117)$. Не наблюдалось статистически достоверного увеличения лучевых реакций со стороны кишечника в 4-й группе относительно других групп.

Таким образом, полученные данные свидетельствуют об улучшении результатов лучевой терапии в условиях СЛТ и АД.

В то же время реализовалась радиобиологическая закономерность: увеличение частоты лучевых поражений при увеличении дозового воздействия, включая как зону опухоли, так и органов риска.

Количественная оценка вероятности лучевых поражений на основе расчетных данных затруднена, учитывая возможное недостаточно совершенное радиобиологическое обоснование прогностических факторов [16]. Поэтому имеет большое практическое значение оценка результатов СЛТ с разной разовой дозой ВДБ по общепринятым критериям: ПСА в динамике, уровень лучевых реакций здоровых тканей $[10,17]$.

\section{Выводы}

- Концентрация ПСА после СЛТ была значительно ниже в группах с АД.

- АД в комплексе с СЛТ при местно-распространенном (Т3а) и локализованном РПЖ с неблагоприятным прогнозом существенно улучшает результаты лечения по критерию биохимического контроля через 2 года после СЛТ: до $91,7 \%, p<0,0087$ (разовая доза брахитерапии 
8,5 Гр) и $100 \%, \mathrm{p}<0,0044$ (разовая доза брахитерапии 9,5 Гр).

- С увеличением дозы брахитерапии с 8,5 до 9,5 Гр (2 имплантации по одной фракции) при СЛТ РПЖ возрастает частота лучевых реакций со стороны мочевыделительной системы, не достигая степеней, вынуждающих прервать лучевое лечение.

\section{Заключение}

В результате проведенных исследований установлено, что эскалация разовой дозы при ВДБ с 8,5 Гр до 9,5 Гр (две имплантации) при СЛТ РПЖ приводила к статистически недостоверному повышению числа пациентов с биохимическим контролем в течение двух лет после лучевой терапии; в тех же условиях при эскалации разовой статистически достоверно возрастала частота ранних лучевых реакций первой и второй степени со стороны мочевыделительной системы, не требующих отмены лучевой тера-

\section{Литература}

1. 25 лет против рака. Успехи и проблемы противораковой борьбы в Беларуси за 1990-2014 годы / А. Е. Океанов [и др.] ; под ред. О. Г. Суконко. - Минск : ГУ РНМБ, 2016. $-415 \mathrm{c}$.

2. Красный, С. А. Результаты скрининга рака предстательной железы и проблема гипердиагностики заболевания в Республике Беларусь / С. А. Красный, Д. Т. Тарендь, С. А. Семёнов // Онкоурология. - 2016. - Т. 12, № 1. - С. 58-62.

3. Овчинников, В. А. Эффективность высокодозной брахитерапии при радикальном лечении рака предстательной железы / В. А. Овчинников, О. С. Довнар // Журнал Гродненского медицинского университета. - 2013. - № 2. - C. $18-23$.

4. Chodak, G.W. Prostate cancer treatment \& management [Electronic resource] / G. W. Chodak // Medscape. - Mode of access: https://emedicine.medscape.com/article/1967731treatment. - Date of access: 14.04.2018.

5. Demanes, D. J. High-dose-rate brachytherapy as monotherapy for prostate cancer / D. J. Demanes, M. I. Chilezan // Brachytherapy. - 2014. - Vol. 13, № 6. - P. 529-541. - doi: 10.1016/j.brachy.2014.03.002.

6. Additional androgen deprivation makes the difference: Biochemical recurrence-free survival in prostate cancer patients after HDR brachytherapy and external beam radiotherapy / J. Schiffmann [et. al.] // Strahlenther. Onkol. - 2015. - Vol. 191, № 4. - P. 330-337.

7. Long-term Follow-up of a Randomized Trial of Radiation With or Without Androgen Deprivation Therapy for Localized Prostate Cancer / A. V. D'Amico [et. al.] // JAMA. - 2015. Vol. 314, № 12. - P. 1291-1293. - doi:10.1001/jama.2015.8577.

8. Does hormone treatment added to radiotherapy improve outcome in locally advanced prostate cancer? : meta-analysis of randomized trials / E. Bria [et. al.] // Cancer. - 2009. - Vol. 115, № 15. - P. 3446-3456. - doi: 10.1002/cncr.24392.

9. Инструкция по применению набора реагентов для иммуноферментного определения общего простатаспецифического антигена в сыворотке (плазме) крови : регистрационное удостоверение № ФСР 2011/11007 от 20 июня 2011 года. - Москва, 2011. - 18 с.

10. Алгоритмы диагностики и лечения больных злокачественными новообразованиями / под ред. О. Г. Суконко, пии, парентерального введения лекарственных средств или хирургического лечения. Указанная эскалация дозы, таким образом, оказалась недостаточной для повышения эффективности СЛТ. Вместе с тем приемлемый уровень лучевых реакций позволяет рассматривать возможность дальнейшего повышения дозы ВДБ.

АД существенно и статистически достоверно улучшала результаты СЛТ с ВДБ РПЖ - локализованный рак с неблагоприятным прогнозом и местно-распространенный (Т3a) по критерию двухлетнего биохимического контроля уровня ПСА. Это повышение эффективности СЛТ в комплексе с СЛТ наблюдалось как при разовой дозе ВДБ 8,5 Гр, так и при разовой дозе 9,5 Гр, достигая при 9,5 Гр 100\% биохимического контроля уровня ПСА.

Полученные данные позволяют рекомендовать АД в комплексном лечении РПЖ с неблагоприятным прогнозом и местно-распространенного (ТЗа) РПЖ.

С. А. Красного. - Минск : Профессиональные издания, 2012. -508 c.

11. Cox, J. D. Toxicity criteria of the Radiation Therapy Oncology Group (RTOG) and the European Organization for Research and Treatment of Cancer (EORTC) / J. D. Cox, J. Stetz, T. F. Pajak // Int. J. Radiat. Oncol. Biol. Phys. - 1995. - Vol. 31, № 5. - P. 1341-1346. - doi: 10.1016/0360-3016(95)00060-C.

12. Boschloo, R. D. Raised Conditional Level of Significance for the 2x2-table when Testing the Equality of Two Probabilities / R. D. Boschloo // Statistica Neerlandica. - 1970. - Vol. 24, № 1. - P. 1-9. - doi: 10.1111/j.1467-9574.1970.tb00104.x.

13. Hommel, G. A stagewise rejective multiple test procedure based on a modified Bonferroni test / G. Hommel // Biometrika. - 1988. - Vol. 75, № 2. - P. 383-386. - doi: 10.1093/biomet/75.2.383.

14. Рак предстательной железы / А. Heidenreich [и др.] ; пер. с англ. О. В. Антонова. - Европейская Ассоциация урологов, 2010. - $186 \mathrm{c}$.

15. Ismail, M. Androgen suppression strategies for prostate cancer: is there an ideal approach? / M. Ismail, M. Ferroni, L. G. Gomella // Curr. Urol. Rep. - 2011. - Vol. 12, № 3. P. 188-196. - doi: 10.1007/s11934-011-0178-0.

16. Pathogenesis of normal-tissue side-effects / W. Dörr // Basic clinical radiology / ed.: M. Joiner, A. van der Kogel. - 4th ed. - London, 2009. - P. 169-190.

17. Biochemical control and toxicity after intensitymodulated radiation therapy for prostate cancer / S. L. Liauw [et al.] // Technol. Cancer Res. Treat. - 2009. - Vol. 8, № 3. P. 201-206. - doi: 10.1177/153303460900800304.

\section{References}

1. Okeanov AE, Moiseev AA, Evmenenko AA, Levin LF; Sukonko OG, editor. 25 let protiv raka. Uspekhi i problemy protivorakovoy borby v Belarusi za 1990-2014 gody [25 years contrary cancer. The successes and challenges of cancer control in Belarus for the years 1990-2014]. Minsk: GU RNMB; 2016. 415 p. (Russian).

2. Krasnyj SA, Tarend DT, Semjonov SA. Rezultaty skrininga raka predstatelnoj zhelezy i problema giperdiagnostiki zabolevanija v Respublike Belarus [The results of prostate cancer screening and the problem of the hyperdiagnosis of the disease in the Republic of Belarus]. Onkourologija [Cancer urology]. 2016;12(1):58-62. (Russian). 
3. Ovchinnikov VA, Dovnar OS. Effektivnost vysokodoznoy brakhiterapii pri radikalnom lechenii raka predstatelnoy zhelezy [Efficiency of high-dose-rate brachytherapy in the radical treatment of prostate cancer]. Zhurnal Grodnenskogo gosudarstvennogo medicinskogo universiteta [Journal of the Grodno State Medical University]. 2013;2:18-23. (Russian).

4. Chodak GW. Prostate cancer treatment \& management [Internet]. Available from: https://emedicine.medscape.com/ article/1967731-treatment.

5. Demanes DJ, Chilezan MI. High-dose-rate brachytherapy as monotherapy for prostate cancer. Brachytherapy. 2014;13(6):529-541. doi: 10.1016/j.brachy.2014.03.002.

6. Schiffmann J, Lesmana H, Tennstedt P, Beyer B, Boehm K, Platz V, Tilki D, Salomon G, Petersen C, Krüll A, Graefen M, Schwarz R. Additional androgen deprivation makes the difference: Biochemical recurrence-free survival in prostate cancer patients after HDR brachytherapy and external beam radiotherapy. Strahlenther. Onkol. 2015;191(4):330-337. doi: 10.1007/ s00066-014-0794-y.

7. D'Amico AV, Chen MH, Renshaw A, Loffredo M, Kantoff PW. Long-term Follow-up of a Randomized Trial of Radiation With or Without Androgen Deprivation Therapy for Localized Prostate Cancer. JAMA. 2015;314(12):1291-1293.

8. Bria E, Cuppone F, Giannarelli D, Milella M, Ruggeri EM, Sperduti I, Pinnarò $\mathrm{P}$, Terzoli E, Cognetti $\mathrm{F}$, Carlini P. Does hormone treatment added to radiotherapy improve outcome in locally advanced prostate cancer?: meta-analysis of randomized trials. Cancer. 2009;115(15):3446-3456. doi: 10.1002/cncr.24392.

9. Instrukcija po primeneniju nabora reagentov dlja immunofermentnogo opredelenija obshhego prostataspecificheskogo antigena v syvorotke (plazme) krovi. RU № FSR 2011/11007.
2011 Ijun 20. Moskva; 2011. 18 p. (Russian).

10. Skonko OG, Krasnyj SA, editors. Algoritmy diagnostiki i lechenija bolnyh zlokachestvennymi novoobrazovanijami. Minsk: Professionalnye izdanija; 2012. 508 p. (Russian).

11. Cox JD, Stetz J, Pajak TF. Toxicity criteria of the Radiation Therapy Oncology Group (RTOG) and the European Organization for Research and Treatment of Cancer (EORTC). Int. J. Radiat. Oncol. Biol. Phys. 1995;31(5):1341-1346. doi: 10.1016/0360-3016(95)00060-C.

12. Boschloo RD. Raised Conditional Level of Significance for the 2x2-table when Testing the Equality of Two Probabilities. Statistica Neerlandica. 1970;24(1):1-9. doi: 10.1111/j.14679574.1970.tb00104.x.

13. Hommel G. A stagewise rejective multiple test procedure based on a modified Bonferroni test. Biometrika. 1988;75(2):383-386. doi: 10.1093/biomet/75.2.383.

14. Heidenreich A, Bolla M, Joniau S, Mason MD, Matveev V, Mottet N, Schmid H-P, van der Kwast TH, Wiegel T, Zattoni F. Rak predstatelnoj zhelezy [Guidelines on Prostate Cancer]. European Association of Urology; 2010. 168 p. (Russian).

15. Ismail M, Ferroni M, Gomella LG. Androgen suppression strategies for prostate cancer: is there an ideal approach? Curr. Urol. Rep. 2011;12(3):188-196. doi: 10.1007/s11934011-0178-0.

16. Dörr W. Pathogenesis of normal-tissue side-effects. In: Joiner M, van der Kogel A, editors. Basic clinical radiology. 4th ed. London: Hodder Arnold; 2009. p. 169-190.

17. Liauw SL, Weichselbaum RR, Rash C, Correa D, AlHallaq HA, Pelizzari CA, Jani AB. Biochemical control and toxicity after intensity-modulated radiation therapy for prostate cancer. Technol. Cancer Res. Treat. 2009;8(3):201-206. doi: $10.1177 / 153303460900800304$.

\title{
COMBINED RADIATION THERAPY FOR PROSTATE CANCER WITH DOSE ESCALATION BRACHYTHERAPY AND ANDROGEN DEPRIVATION
}

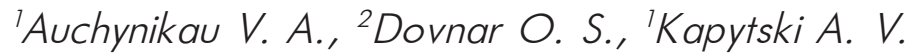

'Educational Institution "Grodno State Medical University», Grodno Belarus

${ }^{2}$ Healthcare Institution "Grodno Regional Clinical Hospital", Grodno, Belarus

\footnotetext{
Background. The efficacy of combined radiation therapy with high dose rate brachytherapy in prostate cancer remains poorly understood.

Purpose of the study: To evaluate the effectiveness of combined radiotherapy of prostate cancer and androgen deprivation in the context of a single-dose escalation brachytherapy (8.5 Gy and $9.5 \mathrm{~Gy}$ ).

Material and methods. We observed 75 patients with locally advanced (T3a) and localized prostate cancer with unfavorable prognosis.

Results. Dose escalation brachytherapy from $8.5 \mathrm{~Gy}$ to $9.5 \mathrm{~Gy}$ (two implants) and androgen deprivation enhance the results of radiation therapy: two-year biochemical control was 100\%.

Conclusion. The effectiveness of treatment of prostate cancer increases with dose escalation radiation therapy and androgen deprivation.

Keywords: combined radiation therapy, prostate cancer, androgen deprivation.
} 